\title{
OXIDATIVE STRESS MARKERS IN ACUTE MYOCARDIAL INFARCTION TREATED BY PRIMARY PERCUTANEOUS CORONARY INTERVENTION
}

\author{
DAN-ALEXANDRU TĂTARU ${ }^{a, b}$, DAN-MIRCEA OLINIC ${ }^{a, b}$ *, \\ ANDRADA URDĂ ${ }^{a}$, MARIA OLINIC ${ }^{\mathrm{a}, \mathrm{b}}$, REMUS ORĂSANa
}

\begin{abstract}
The current study analysed the dynamics of oxidative stress markers in patients with acute ST-elevation myocardial infarction treated by primary percutaneous intervention. Excessive reactive oxygen species production is known to induce myocardial reperfusion injury. There are few studies that evaluated oxidative stress markers in the interventional era, the historical papers were all based on thrombolysis as a mean of reperfusion. Thirty-seven patients were included. Peripheral venous blood samples were obtained prior to coronary angioplasty, at 1 hour and 24 hours after that. Plasma malondialdehyde, reduced glutathione / oxidised glutathione ratio and total antioxidant capacity were determined. Malondialdehyde was significantly lower at 1 hour $(3.1 \pm 0.96 \mathrm{nmol} / \mathrm{ml}$ vs $2.68 \pm 0.81 \mathrm{nmol} / \mathrm{ml}$, $\mathrm{p}<0.01)$ and 24 hours $(3.1 \pm 0.96 \mathrm{nmol} / \mathrm{ml}$ vs $2.15 \pm 0.95 \mathrm{nmol} / \mathrm{ml}, \mathrm{p}<0.01)$. Also, reduced glutathione / oxidised glutathione ratio dropped significantly at 1 hour (3.25 Q1-Q3 2.17-5.19 vs 2.33 Q1-Q3 1.53-2.82, $\mathrm{p}<0.01$ ) and at 24 hours (3.25 Q1-Q3 2.17-5.19 vs 1.96 Q1-Q3 1.28-2.85, $\mathrm{p}<0.01$ ). Total antioxidant capacity had non-significant variation. There was no correlation between these markers and time from symptom-onset or left ventricular ejection fraction. Reperfusion of the occluded coronary artery by percutaneous coronary intervention in acute myocardial infarction led to a rapid decrease of reduced glutathione / oxidised glutathione ratio, that may indicate a depletion of antioxidants as a consequence of overproduction of reactive oxygen species in the damaged area. However, the malondialdehyde level significantly decreased after vessel opening. This may suggest low reperfusion injury after angioplasty.
\end{abstract}

Keywords: Oxidative stress, acute myocardial infarction, malondialdehyde, glutathione, total antioxidant capacity

\footnotetext{
a Iuliu Hațieganu University, Faculty of General Medicine, 8 Victor Babeș street, RO-400012, Cluj-Napoca, Romania

${ }^{b}$ Cluj County Emergency Hospital, Interventional Cardiology Department, 3-5 Clinicilor street, RO-400006, Cluj-Napoca, Romania

*Corresponding author: dolinic@yahoo.com
} 


\section{INTRODUCTION}

Cardiovascular diseases (CVD) are the leading cause of death globally [1]. Myocardial infarction and stroke account for $80 \%$ of CVD deaths in males and $75 \%$ in females [2]. The principal objective of therapy for acute STelevation myocardial infarction (STEMI) is timely restoration of coronary blood flow, to preserve as much heart muscle as possible and to prevent further complications. This can be done by thrombolysis or percutaneous coronary intervention $(\mathrm{PCl})$. However, a sudden restoration of oxygen supply to a previously ischemic myocardium can lead to myocardial reperfusion injury [3], that can paradoxically reduce the beneficial effect of vessel opening. This culminates with the death of cardiac myocytes that were viable immediately before myocardial reperfusion, thus leading to an increase in infarct size.

The mechanism of myocardial reperfusion injury is not fully understood and it is most probably multifactorial: oxidative stress, lipid peroxidation [3], inflammation, microvascular obstruction [4] and excessive cathecolamine release [5].

Malondialdehyde (MDA), a stable lipid peroxidation end-product, is frequently used as a marker of oxidative stress and reactive oxygen species (ROS) production [6].

Reduced glutathione (GSH) is an important antioxidant serving as an electron donor and thereby preventing damage to important cellular components caused by ROS. Oxidised glutathione, also known as glutathione disulphide (GSSG), is a compound that gives information about the usage of GSH during oxidative stress reactions [7]. In a resting cell, the molar GSH: GSSG ratio exceeds 100:1, while in various models of oxidative stress this ratio has been demonstrated to decrease to values of 10:1 and even 1:1 [8].

In contrast to Folin-Ciocâlteu method which is used only 'in vitro' [9], the scavenging of the 1,1-diphenyl-2-picrylhydrazyl (DPPH) free radical offers a simple approach for evaluating the 'in vivo' total antioxidant capacity (TAC) of biological sources [10].

The aim of the current study was to determine the dynamics of MDA, GSH/GSSG ratio and TAC (before reperfusion, 1 hour and 24 hours after angioplasty) in patients admitted with STEMI undergoing emergency successful primary $\mathrm{PCl}$ of the infarct-related artery and study the correlation with the time from symptom-onset and left ventricular ejection fraction (LVEF). The variation of oxidative stress markers was statistically evaluated using ttest for paired samples or Wilcoxon Signed Ranks Test, depending on the variable distribution and Pearson's coefficient to determine correlations. 
According to the authors' knowledge, no comprehensive work was dedicated to oxidative stress markers in acute myocardial infarction treated with primary $\mathrm{PCl}$. The historical papers were all based on thrombolysis as a mean of reperfusion. These studies have showed that, after successful thrombolysis, there is an increase in plasma MDA levels [11-14] and a decrease in antioxidant capacity [11,15]. In the invasive era, $\mathrm{PCl}$ is the preferred reperfusion strategy in infarct-related arteries, since it reduces mortality, as compared to thrombolysis, provided it can be performed expeditiously by an experienced team [16].

\section{RESULTS AND DISCUSSION}

Thirty-seven patients were enrolled. The measured variables of the study group are depicted in Table 1.

The mean MDA concentration before $\mathrm{PCl}\left(\mathrm{P}_{0}\right)$ was $3.1 \pm 0.96 \mathrm{nmol} / \mathrm{ml}$. After reperfusion, a significant decrease of MDA levels occurred at 1 hour $\left(P_{1}\right.$, $2.68 \pm 0.81 \mathrm{nmol} / \mathrm{ml}, \mathrm{p}<0.01)$, and 24 hours $\left(P_{24}, 2.15 \pm 0.95 \mathrm{nmol} / \mathrm{ml}, p<0.01\right)$. Also, levels at 24 hours were significantly lower than MDA concentrations noted at 1 hour, $p<0.01$ (Figure 1 ).

The median GSH/GSSG ratio before reperfusion $\left(P_{0}\right)$ was 3.25 5.19). A significant decrease was observed after 1 hour following $P C l\left(P_{1}, 2.33\right.$ (1.53-2.82), $p<0.01)$ and at 24 hours $\left(P_{24}, 1.96(1.28-2.85), p<0.01\right)$. Also, the difference between $\mathrm{P}_{1}$ and $\mathrm{P}_{24}$ was significant, 2.33 (1.53-2.82) vs 1.96 (1.282.85), $p=0.036$ (Figure 2).

TAC did not have significant variations ( $p=$ non-significant): before reperfusion $\left(P_{0}, 32.7 \pm 4.86\right.$ inhibition \%); at 1 hour after vessel opening $\left(P_{1}, 32.08 \pm 6.42\right.$ inhibition \%); at 24 hours ( $\mathrm{P}_{24}, 33.04 \pm 8.34$ inhibition \%) (Figure 3).

There was no correlation between MDA, GSH/GSSG ratio, TAC levels and the duration of coronary occlusion, as measured by the time from symptom-onset to the opening of the infarct-related artery. Also, no correlation was found between these markers and LVEF at admission. 
Table 1. Variables of the study group. (TSR = time from symptom-onset to reperfusion (hours), LVEF = left ventricular ejection fraction $(\%), \mathrm{MDA}=$ malondialdehyde $(\mathrm{nmol} / \mathrm{ml})$, $\mathrm{G} / \mathrm{G}=$ reduced glutathione / oxidised glutathione ratio, $\mathrm{TAC}=$ total anitioxidant capacity (inhibition \%))

\begin{tabular}{|c|c|c|c|c|c|c|c|c|c|c|c|}
\hline & & & & P0 & & & P1 & & & P24 & \\
\hline & TSR & LVEF & MDA & G/G & TAC & MDA & G/G & TAC & MDA & G/G & TAC \\
\hline Pt 1 & 8 & 35 & 1.94 & 7.96 & 37.61 & 2.09 & 2.33 & 26.03 & 1.66 & 1.56 & 26.66 \\
\hline Pt 2 & 6 & 50 & 1.59 & 8.38 & 37.63 & 1.64 & 4.88 & 40.25 & 2.22 & 4.95 & 39.71 \\
\hline $\mathrm{Pt} 3$ & 3 & 45 & 2.82 & 5.44 & 24.33 & 3.10 & 1.73 & 24.20 & 2.49 & 2.38 & 24.07 \\
\hline $\mathrm{Pt} 4$ & 12 & 40 & 4.71 & 3.10 & 27.06 & 3.87 & 1.21 & 26.32 & 4.23 & 1.43 & 28.41 \\
\hline Pt 5 & 6 & 45 & 3.02 & 2.69 & 41.86 & 3.67 & 1.04 & 49.15 & 3.94 & 1.15 & 58.22 \\
\hline Pt 6 & 2 & 50 & 4.02 & 5.02 & 29.09 & 3.01 & 2.98 & 32.90 & 3.77 & 0.79 & 35.61 \\
\hline Pt 7 & 3 & 50 & 3.48 & 2.31 & 39.13 & 3.20 & 1.88 & 37.49 & 1.54 & 1.79 & 41.74 \\
\hline Pt 8 & 6 & 30 & 2.19 & 3.81 & 26.19 & 1.17 & 4.06 & 27.85 & 0.75 & 3.05 & 28.98 \\
\hline Pt 9 & 2 & 50 & 3.45 & 3.58 & 28.84 & 4.35 & 2.53 & 18.48 & 2.83 & 2.65 & 12.80 \\
\hline Pt 10 & 12 & 50 & 3.30 & 6.35 & 31.26 & 2.17 & 2.34 & 31.68 & 0.53 & 1.25 & 41.93 \\
\hline Pt 11 & 2 & 30 & 2.03 & 8.35 & 38.48 & 1.65 & 4.25 & 41.28 & 0.76 & 3.98 & 42.25 \\
\hline Pt 12 & 5 & 50 & 2.46 & 1.35 & 36.07 & 1.88 & 1.54 & 37.61 & 1.69 & 1.12 & 33.41 \\
\hline Pt 13 & 3 & 35 & 3.16 & 1.25 & 28.38 & 2.28 & 1.35 & 28.02 & 1.32 & 1.05 & 25.72 \\
\hline Pt 14 & 4 & 50 & 2.86 & 2.32 & 24.15 & 2.46 & 2.62 & 22.09 & 2.22 & 1.96 & 21.81 \\
\hline Pt 15 & 5 & 45 & 3.56 & 5.63 & 25.44 & 3.35 & 2.35 & 25.24 & 1.66 & 3.25 & 20.39 \\
\hline Pt 16 & 10 & 40 & 1.96 & 1.65 & 32.38 & 1.72 & 1.36 & 31.45 & 1.06 & 1.98 & 36.40 \\
\hline Pt 17 & 12 & 45 & 4.98 & 3.25 & 25.96 & 3.86 & 2.04 & 25.50 & 3.15 & 1.96 & 27.68 \\
\hline Pt 18 & 4 & 45 & 4.71 & 1.35 & 33.35 & 3.57 & 1.05 & 29.01 & 2.43 & 0.53 & 32.25 \\
\hline Pt 19 & 4 & 50 & 4.06 & 98 & 29 & 2.57 & 1.99 & .13 & 2.03 & 2.02 & .53 \\
\hline Pt 20 & 4 & 50 & 1.96 & 2.63 & 32.88 & 1.92 & 2.02 & 31.48 & 1.21 & 1.25 & 32.64 \\
\hline Pt 21 & 5 & 30 & 2.86 & 2.15 & 35.65 & 2.71 & 1.88 & 37.70 & 1.62 & 1.00 & 35.70 \\
\hline Pt 22 & 4 & 50 & 4.13 & 9.60 & 37.22 & 3.02 & 2.44 & 36.00 & 2.66 & 2.62 & 33.10 \\
\hline Pt 23 & 6 & 50 & 2.80 & 1.48 & 34.30 & 2.08 & 3.99 & 33.45 & 1.93 & 3.26 & 30.52 \\
\hline Pt 24 & 6 & 50 & 3.30 & 1.46 & 39.88 & 3.27 & 1.15 & 30.18 & 3.03 & 1.31 & 29.32 \\
\hline Pt 25 & 5 & 45 & 3.96 & 3.57 & 32.21 & 3.66 & 3.02 & 36.13 & 2.43 & 3.12 & 41.38 \\
\hline Pt 26 & 2 & 50 & 4.53 & 2.25 & 29.64 & 3.80 & 2.39 & 31.05 & 3.34 & 1.36 & 31.60 \\
\hline Pt 27 & 3 & 50 & 2.91 & 4.41 & 36.40 & 2.61 & 0.95 & 33.44 & 2.08 & 1.59 & 32.21 \\
\hline Pt 28 & 4 & 50 & 1.28 & 3.25 & 32.64 & 1.71 & 2.50 & 31.08 & 1.09 & 2.03 & 37.64 \\
\hline Pt 29 & 6 & 45 & 1.48 & 1.65 & 37.06 & 1.29 & 2.02 & 37.38 & 1.52 & 1.02 & 43.41 \\
\hline Pt 30 & 12 & 50 & 3.44 & 2.97 & 27.90 & 2.44 & 8.10 & 20.05 & 2.81 & 3.34 & 22.40 \\
\hline Pt 31 & 3 & 50 & 2.01 & 6.56 & 32.38 & 1.84 & 5.34 & 33.95 & 1.66 & 2.56 & 37.60 \\
\hline Pt 32 & 12 & 50 & 2.69 & 5.35 & 39.81 & 2.40 & 4.35 & 37.85 & 1.37 & 3.25 & 37.42 \\
\hline Pt 33 & 12 & 40 & 4.16 & 4.25 & 36.45 & 3.19 & 2.53 & 31.54 & 2.33 & 1.53 & 41.67 \\
\hline Pt 34 & 6 & 50 & 4.00 & 3.36 & 32.46 & 3.43 & 2.65 & 37.00 & 3.97 & 1.98 & 36.56 \\
\hline Pt 35 & 12 & 40 & 3.28 & 2.18 & 32.97 & 3.06 & 1.98 & 31.57 & 2.70 & 5.66 & 31.10 \\
\hline Pt 36 & 12 & 50 & 2.69 & 4.55 & 27.03 & 2.49 & 0.90 & 26.82 & 2.20 & 1.82 & 21.26 \\
\hline Pt 37 & 12 & 50 & 2.95 & 2.94 & 36.14 & 2.58 & 1.52 & 41.62 & 1.30 & 2.43 & 37.45 \\
\hline
\end{tabular}




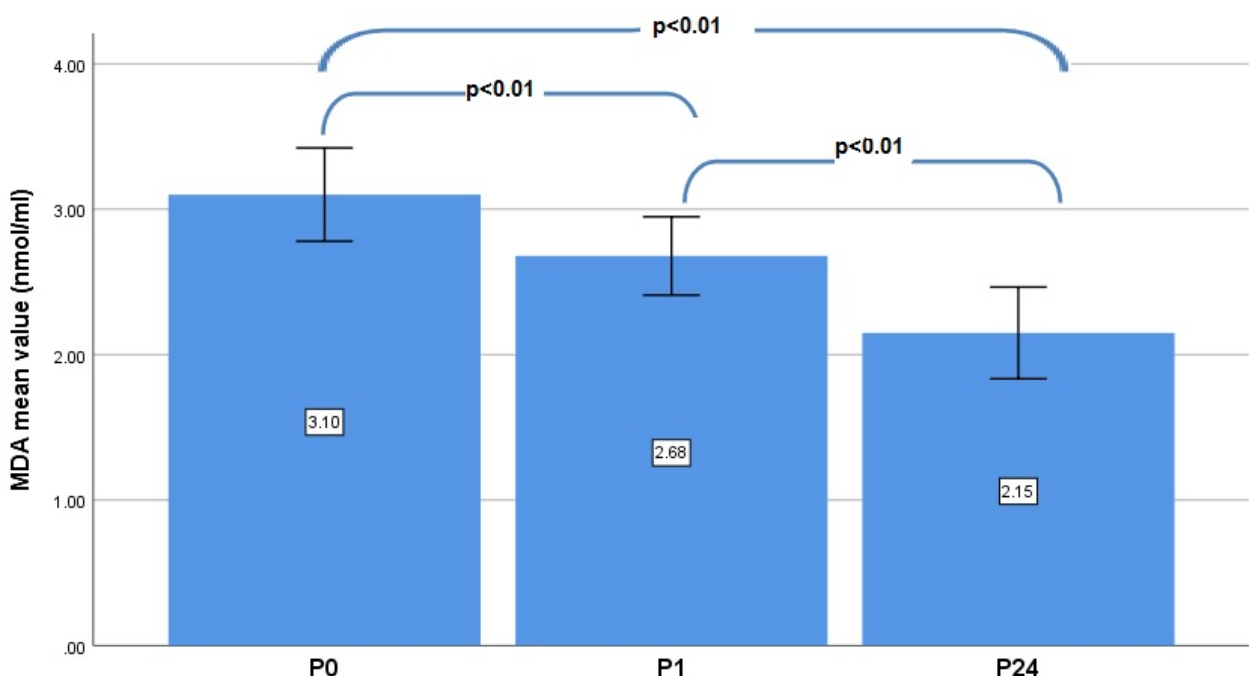

Figure 1. Comparison of MDA mean values: $P_{0}$ - before reperfusion; $P_{1}-1$ hour after $\mathrm{PCl} ; \mathrm{P}_{24}-24$ hours after PCl (t-test for paired samples, error bars: 95\% confidence interval)

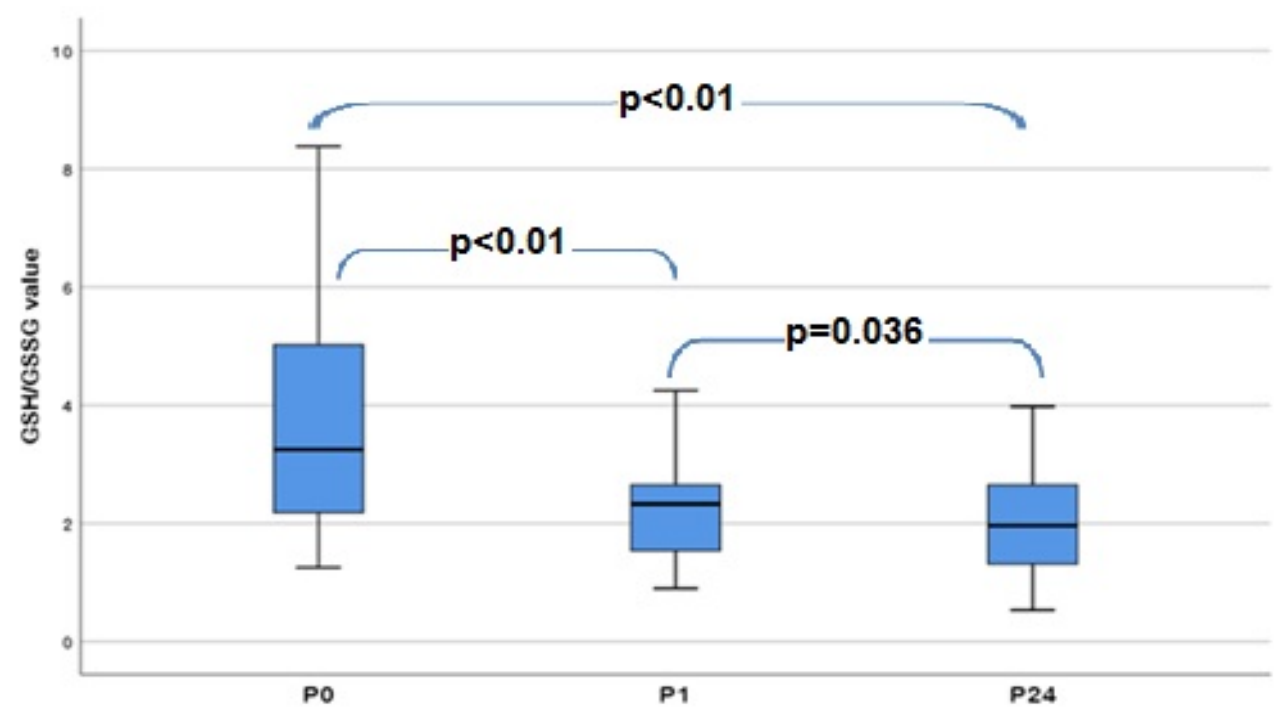

Figure 2. Distribution of GSH/GSSG values; $P_{0}$ - before reperfusion; $P_{1}$ - 1 hour after $\mathrm{PCl} ; \mathrm{P}_{24}-24$ hours after PCl (Wilcoxon Signed Ranks Test; bars = range, box = first quartile to third quartile, horizontal black line $=$ median) 


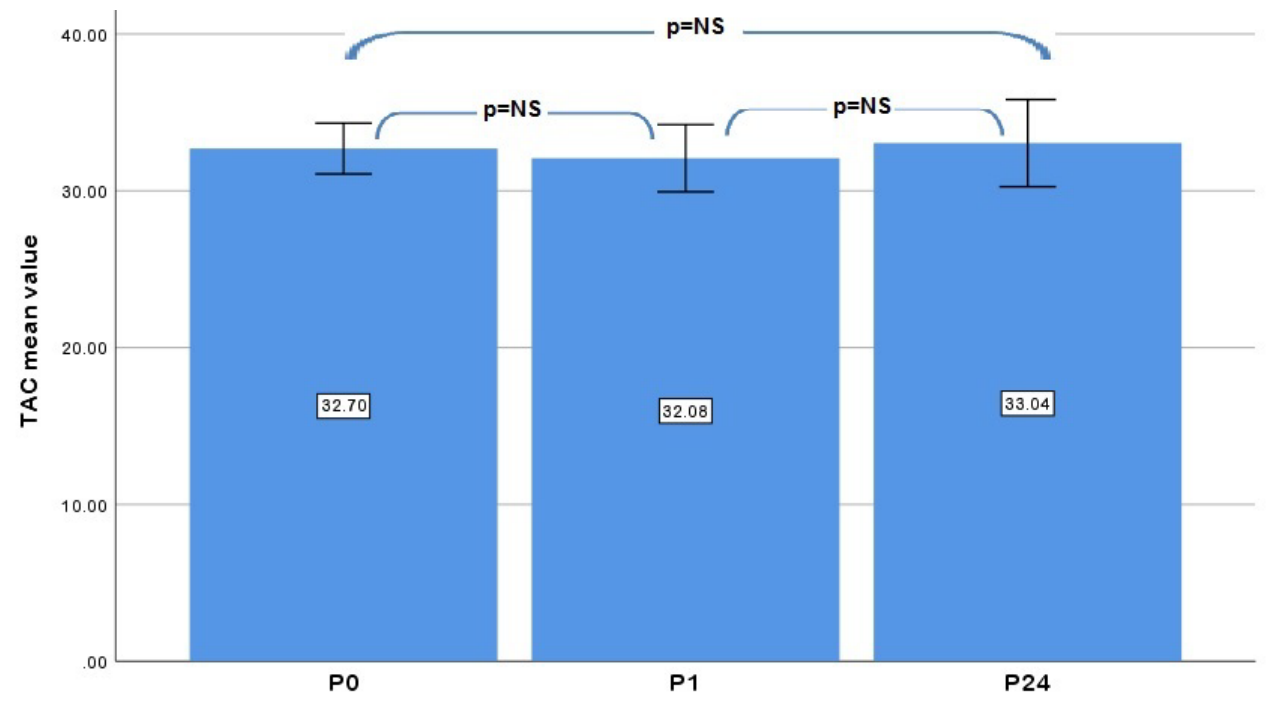

Figure 3. Comparison of TAC mean values; $P_{0}$ - before reperfusion; $P_{1}-1$ hour after $\mathrm{PCl}$; $\mathrm{P}_{24}-24$ hours after $\mathrm{PCl}$ (t-test for paired samples, error bars: 95\% confidence interval; NS = non-significant)

Several studies have evaluated MDA levels in patients with STEMI treated with thrombolytic therapy [10-14]. In all studies, there was an increase of MDA concentrations after thrombolysis, although in some studies, the increase did not reach the level of statistical significance. Two studies $[10,12]$ reported that, after an initial increase, there is a decrease in MDA levels, 2 to 6 hours after successful thrombolysis.

Although a rise in MDA levels was expected, MDA concentrations actually decreased significantly after $\mathrm{PCl}$. Similar findings were reported in other PCl-based studies $[6,17]$. This can be explained by the faster and complete coronary vessel opening after $\mathrm{PCl}$, as compared to thrombolysis. The decrease in MDA supports low lipid peroxidation in myocardial infarction patients treated with primary $\mathrm{PCl}$.

Lipid peroxidation was evaluated by the thiobarbituric acid (TBA) fluorescence method. This approach has potential limitations with respect to specificity. Another way of determining MDA is high-performance liquid chromatography, which is more specific [18]. Considering that the TBA method can detect even non-lipid related, TBA-reactive substances [18], the results suggest that oxidant-mediated injury may actually be modest in $\mathrm{PCl}$ treated patients. The finding of consistently and progressively decreasing MDA levels after reperfusion supports this conclusion. 
GSH/GSSG ratio had a significant decrease after $\mathrm{PCl}$, showing increased oxidative stress and a possible sign of myocardial reperfusion injury, which is in contrast with reduced lipid peroxidation. Similarly, another study reported that human hearts subjected to cardioplegia and subsequent reperfusion show oxidative stress, without an increase in lipid peroxidation [19].

TAC levels had non-significant variations before and after vessel opening. Other authors reported a significant drop in the first hours after $\mathrm{PCl}$ reperfusion, but the levels returned to baseline values on the third day [6]. In the mentioned study [6], TAC levels were assessed by a different method, using the 2,2'-azino-bis(3-ethylbenzthiazoline-6-sulphonic acid) reaction [20].

Although an experimental study on rat hearts reported increased MDA levels during prolongation of ischemia [21], there was no correlation in the present study between oxidative stress markers and the time from symptomonset to reperfusion, which is similar to other $\mathrm{PCl}$ studies [6].

In animal models with acute myocardial infarction, a significant positive correlation between GSH/GSSG ratio and LVEF was observed [22]. In contrast, there was no correlation between LVEF and oxidative stress markers in this study group.

The present study showed that in STEMI patients, $\mathrm{PCl}$ reduces some of the oxidative stress burden, such as lipid peroxidation, while enhacing ROS production by other pathways. This may suggest different mechanisms leading to oxidative stress. Further studies are needed to properly differentiate the pathophysiology of oxidative stress production after reperfusion.

\section{CONCLUSION}

In STEMI patients, the decrease in GSH/GSSG ratio after $\mathrm{PCl}$ suggests increased oxidative stress and is a possible marker of myocardial reperfusion injury.

However, the decrease in MDA levels immediately after vessel opening suggests low oxidative damage after PCl.

There were no significant variations in TAC before and after $\mathrm{PCl}$.

No correlations between MDA, GSH/GSSG ratio, TAC and time from symptom-onset to reperfusion or LVEF were observed.

\section{EXPERIMENTAL SECTION}

The study complied with the Declaration of Helsinki [23]. Written consent was obtained from each patient before the procedure. Patients diagnosed with STEMI and admitted to the Cluj County Emergency Hospital, 
Interventional Cardiology Department were enrolled between April 2017 and December 2017. Inclusion criteria were as follows: electrocardiographic evidence of ST elevation of $\geq 1 \mathrm{mV}$ in two or more standard limb leads or $\geq 2$ $\mathrm{mV}$ in two or more precordial leads; typical chest pain lasting more than 20 min; time of presentation under 12 hours since symptom onset. $\mathrm{PCl}$ was confined to the infarct-related artery and all patients received drug eluting stents. The procedure was considered successful, if the thrombolysis in myocardial infarction (TIMI) flow was 3 and a residual stenosis was under $30 \%$. Only patients with successful reperfusion were included in the study. Patients with Killip class $>3$ (cardiogenic shock) were excluded because of the very severe condition and need of intensive care, which could influence the results. All patients were pretreated with double anti-platelet therapy before $\mathrm{PCl}$ and received $100 \mathrm{UI} / \mathrm{kg}$ of unfractionated heparin during the procedure. Baseline echocardiography before the procedure was performed to assess LVEF and possible complications. All images were stored in the Department's Digital Imaging and Communications in Medicine database [24], for future analysis. The baseline demographic, clinical and non-clinical characteristics are presented in Table 2.

Table 2. Baseline characteristics of the study group. (SD = standard deviation, TSR = time from symptom-onset to reperfusion, $\mathrm{BMI}=$ body-mass index, $\mathrm{PCl}=$ percutaneous coronary intervention, $\mathrm{LAD}=$ left anterior descending artery, $\mathrm{CX}=$ circumflex artery, $\mathrm{RCA}=$ right coronary artery, $\mathrm{LVEF}=$ left ventricular ejection fraction)

\begin{tabular}{|l|l|l|l|}
\hline \multicolumn{5}{|l|}{ Presentation } & \multicolumn{2}{l|}{ Medical history } \\
\hline Age (years), mean \pm SD & $63 \pm 11$ & Previous PCI (yes), no (\%) & $2(5.4)$ \\
\hline Sex (male), no (\%) & $24(64.9)$ & Prior infarction (yes), no (\%) & $5(13.5)$ \\
\hline TSR (hours), mean \pm SD & $6.35 \pm 3.63$ & Infarct-related artery & $18(48.6)$ \\
\hline Killip I (yes), no (\%) & $30(81.1)$ & LAD (yes), no (\%) & $10(27)$ \\
\hline Killip II (yes), no (\%) & $5(13.5)$ & CX (yes), no (\%) & $9(24.4)$ \\
\hline Killip III (yes), no (\%) & $2(5.4)$ & RCA (yes), no (\%) & $45.54 \pm 6.43$ \\
\hline Risk factors & & Echocardiography & \\
\hline Hypertension (yes), no (\%) & $26(70.3)$ & LVEF (\%), mean \pm SD & \\
\hline Dyslipidemia (yes), no (\%) & $23(62.1)$ & & \\
\hline Smokers (yes), no (\%) & $14(37.8)$ & & \\
\hline Diabetes (yes), no (\%) & $7(18.9)$ & & \\
\hline BMI (kg/m2), mean \pm SD & $29.1 \pm 4.3$ & & \\
\hline
\end{tabular}

Peripheral venous blood samples were obtained from each patient immediately before coronarography $\left(P_{0}\right)$ and then after 1 hour $\left(P_{1}\right)$ and 24 hours $\left(\mathrm{P}_{24}\right)$ after successful $\mathrm{PCl}$. Samples were drawn into plastic tubes with 
EDTA. Blood was centrifuged within 1 hour at $1500 \mathrm{rpm}$ for 15 minutes, and the collected plasma was stored at $-30^{\circ} \mathrm{C}$ until analysis, within 28 days. All the reagents were supplied by Sigma (Deisenhofen, Germany).

MDA was determined by the TBA reaction [25]. First, $50 \mu \mathrm{l}$ of plasma was boiled with $1 \mathrm{ml}$ of $10 \mathrm{mM}$ 2-TBA and $1 \mathrm{ml}$ of $75 \mathrm{mM} \mathrm{K}_{2} \mathrm{HPO}_{4}, \mathrm{pH}=3$. After sudden cooling, the product was extracted with n-butanol. Using fluorescence spectrometry (Lambda 35, Perkin Elmer, USA), the sample was measured at an emission wavelength of $534 \mathrm{~nm}$ with a synchronous fluorescence technique at a difference of $14 \mathrm{~nm}$ between the excitation and emission wavelength. The MDA levels were calculated according to a calibration curve realised with known concentrations of MDA.

GSH was determined by fluorescence spectrometry [26]. A $500 \mu \mathrm{l}$ aliquot of plasma is added to $500 \mu \mathrm{l}$ of cold $10 \%$ trichloroacetic acid. After 10 minutes in ice, the mixture is centrifuged (3000 rpm for 15 minutes) and 200 $\mu \mathrm{l}$ of the supernatant is mixed with $1.7 \mathrm{ml}$ of phosphate buffer and $0.1 \mathrm{ml}$ of ophthalaldehyde. After 15 minutes, the fluorescence at $350 \mathrm{~nm}$ excitation and $420 \mathrm{~nm}$ emission is read against a blank (Lambda 35, Perkin Elmer, USA).

For GSSG estimation [27], $250 \mu$ of the plasma sample was incubated with $0,1 \mathrm{ml} 40 \mathrm{nM}$-ethylmaleimide for 30 minutes followed by addition of 0,65 $\mathrm{ml}$ of $0.1 \mathrm{M} \mathrm{NaOH}$. Thereafter, the same procedure was followed for fluorescence development as used in GSH measurement, except in place of the buffer, $0.1 \mathrm{M} \mathrm{NaOH}$ was used.

The concentrations of GSH and GSSG were calculated from calibration curves and are expressed in $\mu \mathrm{mol} / \mathrm{ml}$.

TAC was determined by measurement of DPPH method [28]. The reduction assay was performed by adding $20 \mu \mathrm{l}$ of plasma to $400 \mu \mathrm{l}$ of $0.1 \mathrm{mM}$ methanol solution of $\mathrm{DPPH}$ and phosphate buffer, $\mathrm{pH}=7,4$. After 30-minute incubation at ambient temperature, absorbance of the samples at $520 \mathrm{~nm}$ was measured (Lambda 35, Perkin Elmer, USA) and compared with that of a reference sample containing only DPPH solution and phosphate buffer. TAC was measured in inhibition \% as [(reference extinction - serum extinction) / reference extinction] $\times 100$.

The statistical analysis was performed using SPSS software v25 for Windows (IBM, USA). The Shapiro-Wilk test was used to assess for a normal distribution. The t-test for paired samples was used for normally distributed data and Wilcoxon Signed Ranks Test for paired samples for not normally distributed. The significance threshold was set at $p=0.05$. The data are presented as mean \pm standard deviation for normally distributed samples; otherwise as median and interquartile range $(\mathrm{Q} 1-\mathrm{Q} 3)$, where $\mathrm{Q} 1=$ first quartile and Q3 = third quartile. Correlation analysis was performed using Pearson's correlation coefficient. 


\section{ACKNOWLEDGEMENTS}

We thank our colleagues from The Oxidative Stress Laboratory, part of the Physiology Department, Iuliu Hatieganu University, who provided insight and expertise that greatly assisted the research.

\section{REFERENCES}

1. D. Olinic, M. Spinu, M. Olinic, C. Homorodean, D. Tataru, A. Liew, G. Schernthaner, A. Stanek, G. Fowkes, M. Catalano, International Angiology, 2018, 37, 327.

2. A.S. Go, D. Mozaffarian, V.L. Roger, Circulation, 2013, 127, e6.

3. D. Yellon, D. Hausenloy, New England Journal of Medicine, 2007, 357, 1121.

4. M. Marc, A.C. Iancu, C. Ober, C. Homorodean, S. Balanescu, A.V. Sitar, S. Bolboaca, I. M. Dregoesc, Scientific Reports, 2018, 8, 1897.

5. C. Homorodean, M. Ober, M. Olinic, R. Homorodean, A. Hassoune, D. Tătaru, M. Spînu, D. Olinic, Medical Ultrasonography, 2016, 18, 475.

6. E. Sedláková, O. Rácz, E. Lovásová, R. Beňačka, M. Kurpas, A. Chmelárová, J. Sedlák, M. Studenčan, Central European Journal of Medicine, 2009, 4, 26.

7. D.M. Mitrea, S. Clichici, A. Filip, D. Olteanu, I. Baldea, R. Moldovan, N. Decea, O.A. Hoteiuc, Studia UBB Chemia, 2017, 62, 89.

8. Y. Chai, S. Ashraf, K. Rokutan, R. Johnston, J. Thomas, Archives of Biochemistry and Biophysics, 1994, 310, 273.

9. I. Simon, D. Simedru, L. Dordai, E. Luca, V. Fuss, A. Becze, Studia UBB Chemia, 2016, 61, 505.

10. S.B. Kedare, R.P. Singh, Journal of Food Science and Technology, 2011, 48, 412.

11. T. Beard, D. Carrie, M. Boyer, B. Boudjemaa, J. Ferrières, M. Delay, Archives des maladies du coeur et des vaisseaux, 1994, 87, 1289.

12. K. Iqbal, M.A. Rauoof, M. Mir, N. Tramboo, J. Malik, B. Naikoo, American Journal of Cardiology, 2002, 89, 334.

13. I. Young, J. Purvis, J. Lightbody, A. Adgey, European Heart Journal, 1993, 14, 1027.

14. S. Pucheu, C.H. Coudray, G. Vanzetto, A. Favier, J. Machecourt, J. de Leiris, Free Radical Biology and Medicine, 1995, 19, 873.

15. K. Berg, P. Jynge, K. Bjerve, S, Skarra, S. Basu, R. Wiseth, Free Radical Research, 2005, 6, 629.

16. B. Ibanez and the ESC Taskforce, European Heart Journal, 2017, 00, 1.

17. K. Olsson, J. Harnek, A. Ohlin A, N. Pavlidis, B. Thorvinger, H. Ohlin, Scandinavian Cardiovascular Journal, 2002, 36, 237. 
18. C. Ceconi, A. Cargoni, E. Pasini, et al, American Journal of Physiology-Heart and Circulatory Physiology, 1991, 260, H1057.

19. M. Janssen, J.F. Koster, E. Bos, J. De Jong, Circulation Research, 1993, 73, 681.

20. C. Rice Evans, N. Miller, Methods in Enzymology, 1994, 234, 279.

21. B. Tavazzi, D. Di Pierro, M. Bartolini, M. Marino, S. Distefano, M. Galvano, Free Radical Research, 1998, 1, 25.

22. A. Tavares, A. da Rosa Araujo, S. Llesuy, et al, Experimental and Clinical Cardiology, 2012, 17, 263.

23. World Medical Association, Journal of the American Medical Association, 2013, 310, 2191.

24. C. Homorodean, M. Olinic, D.M. Olinic, Medical Ultrasonography, 2012, 14, 29.

25. M. Conti, P.C. Morand, P. Levillain, A. Lemonnier, Clinical Chemistry, 1991, 37, 1273.

26. M.L. Hu, Methods in Enzymology, 1994, 233, 380.

27. P. Vats, V.K. Singh, S.S. Sing, Aviation, Space and Environmental Medicine, 2008, 79, 1106.

28. A. Janaszewska, G. Bartosz, Scandinavian Journal of Clinical and Laboratory Investigation, 2002, 62, 231. 